\title{
HLA Associations and Clinical Implications in T-Cell Mediated Drug Hypersensitivity Reactions: An Updated Review
}

\author{
Chi-Yuan Cheng, ${ }^{1}$ Shih-Chi Su, ${ }^{2}$ Chi-Hua Chen, ${ }^{1}$ Wei-Li Chen, \\ Shin-Tarng Deng, ${ }^{1}$ and Wen-Hung Chung, ${ }^{2,3,4}$ \\ ${ }^{1}$ Department of Pharmacy Administration, Chang Gung Memorial Hospital, Linkou 33305, Taiwan \\ ${ }^{2}$ Department of Dermatology, Drug Hypersensitivity Clinical and Research Center, Chang Gung Memorial Hospitals, \\ Linkou 33305, Taiwan \\ ${ }^{3}$ College of Medicine, Chang Gung University, Taoyuan 33302, Taiwan \\ ${ }^{4}$ Department of Dermatology, Keelung Chang Gung Memorial Hospital, No. 222 Maijin Road, Anle District, \\ Keelung City 20448, Taiwan \\ Correspondence should be addressed to Wen-Hung Chung; chung1@cgmh.org.tw
}

Received 13 February 2014; Accepted 28 March 2014; Published 8 May 2014

Academic Editor: Jose Antonio Cornejo-García

Copyright (C) 2014 Chi-Yuan Cheng et al. This is an open access article distributed under the Creative Commons Attribution License, which permits unrestricted use, distribution, and reproduction in any medium, provided the original work is properly cited.

T-cell mediated drug hypersensitivity reactions may range from mild rash to severe fatal reactions. Among them, drug reaction with eosinophilia and systemic symptoms (DRESS) or drug-induced hypersensitivity syndrome (DIHS), Stevens-Johnson syndrome/ toxic epidermal necrolysis (SJS/TEN), are some of the most life-threatening severe cutaneous adverse reactions (SCARs). Recent advances in pharmacogenetic studies show strong genetic associations between human leukocyte antigen (HLA) alleles and susceptibility to drug hypersensitivity. This review summarizes the literature on recent progresses in pharmacogenetic studies and clinical application of pharmacogenetic screening based on associations between SCARs and specific HLA alleles to avoid serious conditions associated with drug hypersensitivity.

\section{Introduction}

Severe cutaneous adverse reactions to drugs (SCARs) include syndromes such as Stevens-Johnson syndrome/toxic epidermal necrolysis (SJS/TEN) and drug reaction with eosinophilia and systemic symptoms (DRESS) or druginduced hypersensitivity syndrome (DIHS) $[1,2]$. Although the incidence of SJS and TEN is very low $(0.4-6$ cases per million persons per year), they are severe, life-threatening adverse drug reactions with mortality rates as high as $5 \%$ to $12.5 \%$ for SJS and $50 \%$ for TEN $[1,3]$. In patients with DRESS, skin rash is usually associated with fever, hepatitis and/or other internal organ involvement, lymphadenopathy, and hematological abnormalities (leukocytosis, eosinophilia, and atypical lymphocytosis). DRESS is also a rare adverse drug reaction and often has a relapsing-remitting course despite withdrawal of the drug. Reactivation of human herpesvirus
(HHV), mainly HHV-6 and less frequently cytomegalovirus, has been described during the course of DRESS [4-7]. These viral reactivations have been reported in association with recurrence of symptoms more than 2 weeks after the drug was discontinued $[5,6]$. SCARs are generally considered unpredictable irrespective of dosage.

In this decade, an important progress for the underlying mechanisms of SCARs has been achieved by the discovery of association between HLA alleles and SCARs. In 2004, we first reported a strong association of HLA-B ${ }^{*} 15: 02$ with carbamazepine-induced SJS/TEN [8]. More importantly, we found that HLA-B* $15: 02$ is specific for the carbamazepineinduced activation of cytotoxic T lymphocytes (CTLs) in SIS/TEN patients. Upon the stimulation, CTLs expressed a large amount of granulysin which was identified as a key mediator responsible for the extensive epidermal necrolysis in SJS/TEN [9]. 
TABLE 1: Associations of SCARs and delayed-type drug hypersensitivity and HLA alleles.

\begin{tabular}{|c|c|c|c|c|c|}
\hline Causative drug & HLA allele & Hypersensitivity reactions & Ethnicity & Odds ratio $(95 \% \mathrm{CI})$ & Reference \\
\hline Abacavir & $\mathrm{B}^{*} 57: 01$ & Abacavir hypersensitivity & Caucasians & $117(29-481)$ & {$[48]$} \\
\hline \multirow[t]{2}{*}{ Allopurinol } & $\mathrm{B}^{*} 58: 01$ & SJS/TEN/DRESS & Asians & $74.18(26.95-204.14)$ & {$[34]$} \\
\hline & & & Non-Asians & $101.45(44.98-228.82)$ & {$[34]$} \\
\hline \multirow[t]{17}{*}{ Carbamazepine } & $B^{*} 15: 02$ & SJS/TEN & Han Chinese & $115.32(18.17-732.13)$ & {$[17]$} \\
\hline & & & Thai & $54.43(16.28-181.96)$ & {$[17]$} \\
\hline & & & Malaysians & $221.00(3.85-12694.65)$ & {$[17]$} \\
\hline & & & Indians & $54.60(2.25-1326.20)$ & {$[17]$} \\
\hline & $\mathrm{B}^{*} 15: 08$ & & Indians & nd & {$[15]$} \\
\hline & $\mathrm{B}^{*} 15: 11$ & & Japanese & $16.3(4.76-55.61)$ & {$[23]$} \\
\hline & & & Koreans & $18.0(2.3-141.2)$ & {$[22]$} \\
\hline & & & Mainland China Han Chinese & $31.00(2.74-350.50)$ & {$[24]$} \\
\hline & $\mathrm{B}^{*} 15: 18$ & & Japanese & $13.58(\mathrm{nd})$ & {$[25]$} \\
\hline & $A^{*} 31: 01$ & DRESS & Han Chinese & $23.0(4.2-125)$ & {$[20]$} \\
\hline & & & Europeans & $57.6(11.0-340)$ & {$[20]$} \\
\hline & & SJS/TEN & Europeans & $4.4(1.1-17.3)$ & {$[20]$} \\
\hline & & & All populations & $3.94(1.4-11.5)$ & {$[20]$} \\
\hline & & SJS/TEN & Europeans & $25.93(4.93-116.18)$ & {$[19]$} \\
\hline & & DRESS & Europeans & $12.41(1.27-121.03)$ & {$[19]$} \\
\hline & & MPE & Europeans & $8.33(3.59-19.36)$ & {$[19]$} \\
\hline & & SIS/TEN/DRESS & Japanese & $10.8(5.9-19.6)$ & {$[18]$} \\
\hline Dapsone & $\mathrm{B}^{*} 13: 01$ & DRESS & Mainland China Han Chinese & $20.53(11.55-36.48)$ & {$[61]$} \\
\hline Lamotrigine & $\mathrm{B}^{*} 15: 02$ & SIS/TEN & Han Chinese & $3.59(1.15-11.22)$ & {$[27]$} \\
\hline \multirow[t]{4}{*}{ Nevirapine } & $\mathrm{DRB1}^{*} 01: 01$ & DRESS/MPE & Australians & depend on CD4 T-cells count & {$[56]$} \\
\hline & $\mathrm{B}^{*} 14: 02$ & & Sardinians & nd & {$[58]$} \\
\hline & $B^{*} 35: 05$ & & Thai & $18.96(4.87-73.44)$ & {$[57]$} \\
\hline & Cw8 & & Sardinians, Japanese & nd & {$[58,59]$} \\
\hline Oxcarbazepine & $\mathrm{B}^{*} 15: 02$ & SJS/TEN & Taiwan Han Chinese & $80.7(3.8-1714.4)$ & {$[30]$} \\
\hline Phenytoin & $\mathrm{B}^{*} 15: 02$ & SJS/TEN & Han Chinese & $4.26(1.93-9.39)$ & {$[27]$} \\
\hline
\end{tabular}

DRESS: drug reaction with eosinophilia and systemic symptoms.

MPE: maculopapular eruption.

SCARs: severe cutaneous adverse reactions to drugs.

SIS: Stevens-Johnson syndrome.

TEN: toxic epidermal necrolysis.

CI: confidence interval.

nd: no data.

In this review, we summarize recent progresses in identifying genetic links for SCARs or drug-induced hypersensitivity reactions, related to carbamazepine and other aromatic anticonvulsants (phenytoin, oxcarbazepine, and lamotrigine), allopurinol, antiretroviral drugs (abacavir, nevirapine), and dapsone. Reported findings were summarized and listed in Table 1.

\section{Pharmacogenetics of SCARs and Delayed-Type Drug Hypersensitivity}

\subsection{HLA Alleles Associated Drug Hypersensitivity}

\subsubsection{Carbamazepine and Other Aromatic Amine} Anticonvulsants.

(1) Carbamazepine. Carbamazepine (CBZ), an aromatic amine anticonvulsant, is approved by the US Food and
Drug Administration for the treatment of epilepsy and other seizure disorders, trigeminal neuralgia, and bipolar disorder. Approximately $10 \%$ of patients develop mild cutaneous adverse reactions, for example, maculopapular eruption (MPE), within the first 3 months of CBZ therapy [10], and the incidence of CBZ-induced SJS/TEN is very low (incidence $0.23 \%$ in Taiwan) [11]. In addition, long-term use of CBZ may increase risk of lupus, mostly in women [12].

Phenotype and Ethnicity Specific Association with Specific HLA Alleles of CBZ-Induced SCARs. The most striking evidence of genetic susceptibility to SJS/TEN was provided by our findings that HLA- $B^{*} 15: 02$ is strongly associated with CBZinduced SJS/TEN [8]. We also found that the HLA association is phenotype-specific in CBZ-induced cutaneous reactions in Taiwan, as HLA-B ${ }^{*} 15: 02$ is only associated with SJS/TEN, and HLA-A*31:01 is only associated with MPE and DRESS 
[13]. A following study in Hong Kong Han Chinese also found that the presence of HLA-B ${ }^{*}$ 15:02 greatly increased the risk of CBZ-induced SJS/TEN but not MPE or DRESS [14]. The association of CBZ-induced SJS/TEN and HLA$B^{*}$ 15:02 was subsequently reported in China, as well as in other South/Southeast Asian areas including Thailand, Malaysia, Indian, Vietnam, Cambodia, and Reunion Islands [15]. Recently, systematic reviews and meta-analyses verified strong relationship between the HLA-B ${ }^{*}$ 15:02 allele and CBZinduced SJS/TEN in Han Chinese and certain Southeast Asian populations $[16,17]$. However, the HLA-B ${ }^{*}$ 15:02 allele has not been observed in cases of CBZ-induced SJS/TEN in other ancestral groups such as Japanese and Korean populations or non-Asian descendants in Europe or North America [15]. These observations may be explained by the various HLA allele frequencies in the different populations in the world. The allele frequency of HLA-B* 15:02 among residents of Southeast Asian countries was reported to be 1$8 \%$; however, it is absent or lower than $0.4 \%$ in Europeans or Northeast Asians (e.g., Japan and Korea) [15]. By comparison, Ozeki et al. and McCormack et al. showed that HLA-A*31:01 is the main genetic determinant for all types of CBZ-induced adverse cutaneous reactions, including SJS, TEN, MPE, and DRESS in the Japanese and Europeans [18, 19]. However, there were only very few cases of CBZ-induced SJS/TEN which are included in these analyses. Recently, a follow-up study with a larger sample size from Taiwan and RegiSCAR showed that HLA-A*31:01 was strongly associated with CBZ-induced DRESS in the Europeans (OR 57.6; 95\% CI 11.0-340; $P<$ 0.001 ) as well as in Han Chinese (OR 23.0; 95\% CI 4.2-125; $P<0.001$ ), but HLA-A*31:01 had no association with CBZinduced SJS/TEN in Han Chinese and the Europeans [20]. A meta-analysis confirmed that in all populations, HLA-A* $31: 01$ had a strong association with CBZ-induced DRESS (OR 13.2; 95\% CI 8.4-20.8; $P<0.001$ ), but a much weaker association with CBZ-induced SJS/TEN (OR 3.94; 95\% CI 1.4-11.5; $P=$ 0.01 ) [20]. This finding suggested that HLA-A*31:01 may not be a clinically useful marker for preventing life-threatening SJS/TEN induced by CBZ.

Furthermore, our more recent study with increased sample size (112 CBZ-SJS/TEN) showed that the strength of HLA-B ${ }^{*}$ 15:02 association with CBZ-induced SJS/TEN was also related to the clinical severity. CBZ-SJS/TEN showed the strongest association $(100 \%, 25 / 25)$ with the HLA-B ${ }^{*} 15: 02$ allele in SJS/TEN patients if they had more than $5 \%$ body surface area (BSA) skin detachment but lost its $100 \%$ association $(85.1 \%, 74 / 87)$ in SJS with $<5 \%$ BSA detachment [21].

Other HLA Alleles Link to CBZ-Induced SJS/TEN. Some of the cases of CBZ-induced SJS/TEN did not carry the HLA$B^{*} 15: 02$ allele, suggesting that other genetic variants may play a role. HLA-B* 15:02 belongs to HLA-B75. Other members of HLA-B75 serotype have been implicated in CBZ-induced SJS/TEN, such as HLA-B* 15:08 in the Indians [15], HLA$\mathrm{B}^{*} 15: 11$ in the Koreans [22], the Japanese [23], and Han Chinese in China [24], and Taiwan (our unpublished data), and HLA-B ${ }^{*} 15: 18$ in Japanese [25]. The rationale is that these HLA alleles share high amino acid sequence homology that may resemble structural features of HLA-B ${ }^{*}$ 15:02 and thus may be able to trigger a similar cutaneous adverse reaction to CBZ [26].

Several studies have suggested that certain HLA allele may exert a protective effect against CBZ-induced SJS/TEN, as evidence by lower carrier frequencies in cases compared with CBZ-tolerant controls, including HLA-B* 40:01 in Taiwan [13] and Hong Kong [27] and HLA-B* 07:02 in the Caucasians [28]. However, whether these alleles have functional roles for the protective effect needed to be further studied.

Clinical Implications of HLA-B*15:02 Finding for CBZSJS/TEN. Screening for HLA-B* 15:02 has been recommended by the US Food and Drug Administration prior to starting CBZ in patients with Asian ancestry, particularly for those of Southeast Asian ancestry since December 2007. Based on Clinical Pharmacogenetics Implementation Consortium (CPIC) guidelines for carriers of HLA-B* ${ }^{*} 15: 02$, if the patient is CBZ-naïve, no use of CBZ is strongly recommended regardless of ancestry, and if the patient has previously used CBZ for longer than 3 months without incidence of cutaneous adverse reactions, use of CBZ is cautiously considered [10].

The translation of HLA-B* 1502 and CBZ-induced SJS/TEN from discovery to a guideline-based test used routinely in Taiwan is a notable example (Table 2) [29]. Since 2010 in Taiwan, the National Health Insurance has covered the expense of the genetic screening for HLA-B ${ }^{*} 15: 02$ in individuals initiating CBZ. The success of HLA-B* 15:02 screening in reducing the incidence of CBZ-induced SJS/TEN has been demonstrated clinically from 23 hospitals in Taiwan [11]. After applying genetic screening for HLA$B^{*} 15: 02$ prior to initiating CBZ, there was no cases of either SJS or TEN reported. This outcome was significant since approximately 8 cases were expected based on the estimated historical incidence of $0.23 \%$ for CBZ-induced SJS/TEN in Taiwan [11].

(2) Other Aromatic Amine Anticonvulsants (Phenytoin, Oxcarbazepine, and Lamotrigine). Phenytoin, oxcarbazepine, lamotrigine, and CBZ are metabolized to arene oxide metabolites. Our study revealed that HLA-B* $15: 02$ was associated with an increased risk of SJS/TEN upon exposure to phenytoin, oxcarbazepine, and potentially lamotrigine in a Taiwanese population although the strength of these associations was weaker than that of CBZ [30]. Small case studies in Thailand (4 cases phenytoin induced SJS) and Hong Kong (single cases of phenytoin and lamotrigine induced SJS) also showed the presence of HLA-B ${ }^{*}$ 15:02 in all SJS patients [14, 31]. Recently, a meta-analysis of the relationship between aromatic amine anticonvulsants-induced SJS/TEN and HLA-B ${ }^{*}$ 15:02 in Han Chinese populations showed a strong association of HLA$B^{*} 15: 02$ with phenytoin (OR 4.26; 95\% CI 1.93-9.93; $P<3 \times$ $10^{-4}$ ) and, to a lesser extent, with lamotrigine (OR 3.59; 95\% CI 1.15-11.22; $P=0.03$ ) [27]. These studies confirmed a clinically relevant association between the HLA-B ${ }^{*}$ 15:02 allele and phenytoin-induced SJS/TEN, supporting the US Food and Drug Administration recommendation that health care 
TABLE 2: Translational roadmap of CBZ-SJS research and its clinical applications [24].

\section{8-2004}

CBZ, an aromatic anticonvulsant, was recognized as the major cause of SJS, as listed in the records of Taiwan Drug Relief Foundation.

\section{4}

A strong association of CBZ-induced SJS with HLA-B*15:02 in Han Chinese was first uncovered in Taiwan.

\section{6}

The correlation of CBZ-induced SJS with HLA-B $* 15: 02$ was not existent in Caucasian patients, indicating an ethnical specificity of $\mathrm{HLA}-\mathrm{B} * 15: 02$ allele.

2007-2008

The association of CBZ-induced SJS with HLA-B $* 15: 02$ was found among many populations in Southeast Asia.

2007

The US Food and Drug Administration has published an alert to healthcare professionals on the use of CBZ to Asians. (The incidence rate of CBZ-induced SJS is 5.9/10000 in Taiwan and 0.2/10000 in the US.)

2007

The Taiwan and US Food and Drug Administration relabeled the drug information of CBZ and recommended a genetic screening of HLA-B $* 15: 02$ prior to starting CBZ in patients with Asian ancestry, particularly for those of Southeast Asian ancestry.

2010

The National Health Insurance in Taiwan has covered the expense of the genetic screening for HLA-B* 15:02 in individuals initiating CBZ.

CBZ: carbamazepine; SJS: Stevens-Johnson syndrome; HLA: human leukocyte antigen.

providers should consider avoiding phenytoin and its prodrug, fosphenytoin, as alternatives for CBZ in HLA-B* 15:02 carriers (http://www.fda.gov/Drugs/DrugSafety/Postmarket DrugSafetyInformationforPatientsandProviders/ucm110259 .htm).

2.1.2. Allopurinol. Allopurinol is a drug that has been used for decades to lower the levels of serum urate in patients with gout and in cancer patients undergoing chemotherapy at risk of tumor lysis syndrome. The greatest safety concern with allopurinol is an estimated $0.1-0.4 \%$ risk of SCARs [32]. Allopurinol is a major cause of SCARs, and one of the most serious, with up to $25 \%$ mortality [32]. The association of HLA-B ${ }^{*}$ 58:01 with allopurinol-induced SCARs was first identified in Taiwan (our published data) [32]. In our study, HLA-B ${ }^{*} 58: 01$ was present in $100 \%(51 / 51)$ of the patients with allopurinol-induced SCARs, as compared with $15 \%$ (20/153) of allopurinol-tolerant controls and 20\% (19/93) of the population controls [33]. This strong association was replicated in Han Chinese in China [32] and Thai populations [32]. A similar but more modest association was observed in Korean, Japanese, and European populations [32]. One systematic review and meta-analysis that consolidated the published studies showed a strong and significant association between HLA-B ${ }^{*}$ 58:01 and allopurinol-induced SJS/TEN with odds ratio of 74.18 (95\% CI 26.95-204.14) and 101.45 (95\% CI 44.98-228.82) for Asian and non-Asian populations, respectively [34]. Given the high specificity for allopurinolinduced SCARs, allopurinol is contraindicated in patients who have tested positive for HLA-B ${ }^{*}$ 58:01 according to the CPIC guidelines for HLA-B genotype and allopurinol dosing [32].
The prospective studies of HLA-B* 58:01 genotyping for the prevention of allopurinol-induced SCARs have not been published; however, one such study is under way in Taiwan [35]. A cost-effectiveness analysis for prevention of allopurinol-induced adverse drug reaction based on a retrospective study of Taiwan National Health Insurance database from year 2002 to 2011 revealed that the genetic test for HLA-B ${ }^{*}$ 58:01 in high-risk population, defined as patients older than 60 years or patients with renal dysfunction, was expected to recoup the investment in an average of 8.6 years, and the alternative treatment with febuxostat takes only 1.1 years (our unpublished data). The Taiwan Department of Health has updated the labeling for allopurinol to include information on HLA-B ${ }^{*}$ 58:01 (http://www.tdrf.org.tw/ch/03_message/mes_02_main.asp? bull_id=3529), although the US Food and Drug Administration has not. The updated label describes the strong association between HLA-B* 58:01 and allopurinol-induced SCARs in the Han Chinese population and recommends testing for the allele before the use of allopurinol.

Based on the data from the Han Chinese and Thai populations regarding strong associations between HLA-B* 58:01 and allopurinol-induced SCARs, the positive predictive value for $\mathrm{HLA}^{-\mathrm{B}^{*}} 58: 01$ is only $1.5 \%$ and the negative predictive value is $100 \%[33,36]$. Therefore, a large number of patients carrying the allele will not develop SCARs when they receive allopurinol treatment. New genetic factors may be identified in the future to differentiate the HLA-B ${ }^{*}$ 58:01 carriers who are or are not likely to develop SCARs. However, apart from Han Chinese and Thai populations, the absence of HLA-B ${ }^{*}$ 58:01 does not totally eliminate the possibility of developing SCARs, especially in the European population [32]. 
In addition to SCARs, allopurinol therapy is also associated with a 2-3\% incidence of MPE, a mild cutaneous adverse reaction [32]. Strong association between HLA-B ${ }^{*}$ 58:01 and MPE was found in mainland China (OR, 339.00, $P<$ 0.0001) [37], but not in Australia [38]. However, it should be emphasized that given the small number of subjects, further studies with a larger sample size will be necessary to provide a more definite answer. Recently, American College of Rheumatology's new guidelines on gout has recommended patients of Asian backgrounds to consider genetic test for HLA-B* 58:01 before use of allopurinol [39].

Alternative therapies in the setting of gout now are available. Febuxostat was approved by the US Food and Drug Administration in 2009. It is a nonpurine xanthine oxidase inhibitor that is primarily metabolized in the liver to inactive glucuronide and excreted into the urine and bile. Therefore, febuxostat can be used in patients with mildto-moderate renal dysfunction without dosage adjustment. Moreover, it was reported to be tolerated in patients with a history of severe allopurinol hypersensitivity [40]. The launch of febuxostat is a milestone in the treatment of gout, while an alternative is pegloticase. In 2010, the US Food and Drug Administration approved pegloticase as an orphan drug for treating refractory chronic gout patients who were irresponsive or intolerant to other urate-lowering drugs [41]. Patients with chronic kidney disease and positive for HLA$B^{*}$ 58:01 have been shown to be at higher risk of allopurinolinduced SCARs [42]. Hence, choosing those new drugs (e.g., febuxostat) instead of allopurinol is safer in this group of patients.

2.1.3. Abacavir. Abacavir belongs to the family of nucleoside reverse transcriptase inhibitors used for the treatment of HIV-1 infection. Abacavir hypersensitivity syndrome (ABC HSS) differs from DRESS induced by other drugs, occurring at a median value of 9 days after first initiation of the drug [43]. Unlike DRESS associated with other drugs, eosinophilia and hepatitis are uncommon in patients with ABC HSS. Symptoms of ABC HSS are nonspecific and include fever, malaise, gastrointestinal symptoms, and internal organ involvement. Rash tends to be mild-to-moderate, occurring in $70 \%$ of patients with $\mathrm{ABC}$ HSS and often late in the course of disease. The characteristic of the ABC HSS is that symptoms will completely subside within 3 days of discontinuation [44]. Although abacavir has been commonly associated with a drug hypersensitivity syndrome in $8 \%$ of patients who start the drug [43], there have been only two published case reports of SJS/TEN potentially associated with abacavir in the literature despite over 10 years of postmarketing experience $[45,46]$.

In 2002, two groups independently published a strong association between HLA-B* ${ }^{*}$ 7:01 and abacavir hypersensitivity in Australian and British populations [47, 48]. Abacavir-induced hypersensitivity is present at a high frequency only in Caucasians and at a very low frequency in Asian and Black populations $[49,50]$. Because of a considerable rate of false-positive result of HLA-B $* 57: 01$ test, abacavir patch testing was used as a specific test to identify true abacavir hypersensitivity [51-54].
2.1.4. Nevirapine. Nevirapine is a nonnucleoside reverse transcriptase inhibitor, widely prescribed for HIV-1 infection. DRESS occurs in approximately $5 \%$ of those starting nevirapine and typically associated with fever, rash, and hepatitis and SJS/TEN in $0.3 \%$ or less [55].

Different Class I and Class II HLA associations with nevirapine-induced rash and DRESS across different populations have been described. A population-based study from Western Australia revealed an association of the Class II allele HLA-DRB1*01:01 with rash-associated hepatitis in those with a CD4+ $\geqq 25 \%$ [56]. A study in Thailand showed a high frequency of HLA-B* $35: 05$ (17.5\%) in patients with nevirapine-induced rash and DRESS [57]. HLA-Cw8 and HLA-B ${ }^{*}$ 14:02 associations with nevirapine-induced DRESS were also reported in a Sardinian population [58], and a HLACw8 association was noted in a Japanese population [59]. To date, no specific HLA association has been described in nevirapine-induced SJS/TEN. Recently, Ciccacci et al. suggested that genetic variability in a nevirapine-metabolizing cytochrome P450 2B6 can contribute to the susceptibility to nevirapine-induced SJS/TEN in an Italian study [60].

2.1.5. Dapsone. Dapsone is used in the treatment of infections (e.g., leprosy) and chronic inflammatory diseases characterized by the infiltration of neutrophils or eosinophils (e.g., dermatitis herpetiformis). The dapsone-induced DRESS develops in 0.5 to $3.6 \%$ of persons treated with the drug [59]. Recently, it is reported that HLA-B ${ }^{*} 13: 01$ was associated with dapsone-induced DRESS (OR 20.53; 95\% CI 11.55-36.48; $P=$ $6.84 \times 10^{-25}$ ) among patients with leprosy in mainland China [61].

\subsection{Genetic Associations Other Than HLA Alleles in Drug} Hypersensitivity. Apart from HLA alleles association with drug hypersensitivity, contributions of genetic variants of metabolic enzymes in cutaneous adverse drug reactions had been proposed as well. Nevertheless, most of these results were either frustrating or with uncertain till now. We mention sulfonamide and phenytoin as examples. NAT2 allele encoding $\mathrm{N}$-acetyltransferase 2 , a major enzyme in the sulfonamide metabolism. Recently, Sacco et al. found no association of NAT2 coding alleles with sulfonamide hypersensitivity (predominantly cutaneous eruptions) [62]. Cytochrome $\mathrm{P}_{450}$ (CYP) 2C9, a main metabolic enzyme for phenytoin, may be the pathological mechanism for phenytoininduced cutaneous adverse drug reactions. From a Korean study, they found that a heterozygous CYP2C9* 3 variant could play a role in the proportion of patients with phenytoininduced cutaneous adverse drug reactions [63]. However, there were only very few cases included. Further investigations with a larger sample size are warranted.

\section{Conclusions}

Studies presented in this review show the tremendous progress in the area of pharmacogenetics of SCARs in recent years. In Taiwan, personalized medicine has started identifying patients at high risk of SCARs based on pharmacogenomics by using biomarkers, such as HLA-B ${ }^{*} 15: 02$ for 
carbamazepine, HLA- $\mathrm{B}^{*}$ 58:01 for allopurinol, and resulting in the reduction of serious cutaneous adverse drug reactions. However, biomarkers have only been identified for a few, such as abacavir, carbamazepine, and allopurinol. It is likely that further progress will be made by the nationwide or even international well-defined sampling of patients to identify corresponding genetic markers that can predict patients at high risk on the basis of ethnicity and the causative drug.

\section{Conflict of Interests}

The authors declare that there is no conflict of interests regarding the publication of this paper.

\section{References}

[1] J. C. Roujeau and R. S. Stern, "Severe adverse cutaneous reactions to drugs," The New England Journal of Medicine, vol. 331, no. 19, pp. 1272-1285, 1994.

[2] S. Bastuji-Garin, B. Rzany, R. S. Stern, N. H. Shear, L. Naldi, and J.-C. Roujeau, "Clinical classification of cases of toxic epidermal necrolysis, Stevens-Johnson syndrome, and erythema multiforme," Archives of Dermatology, vol. 129, no. 1, pp. 92-96, 1993.

[3] H. L. Chan, R. S. Stern, K. A. Arndt et al., "The incidence of erythema multiforme, Stevens-Johnson syndrome, and toxic epidermal necrolysis: a population-based study with particular reference to reactions caused by drugs among outpatients," Archives of Dermatology, vol. 126, no. 1, pp. 43-47, 1990.

[4] T. Shiohara, M. Inaoka, and Y. Kano, "Drug-induced hypersensitivity syndrome(DIHS): a reaction induced by a complex interplay among herpesviruses and antiviral and antidrug immune responses," Allergology International, vol. 55, no. 1, pp. $1-8,2006$.

[5] Y. Kano, K. Hiraharas, K. Sakuma, and T. Shiohara, "Several herpesviruses can reactivate in a severe drug-induced multiorgan reaction in the same sequential order as in graft-versus-host disease," British Journal of Dermatology, vol. 155, no. 2, pp. 301306, 2006.

[6] M. Tohyama, K. Hashimoto, M. Yasukawa et al., "Association of human herpesvirus 6 reactivation with the flaring and severity of drug-induced hypersensitivity syndrome," British Journal of Dermatology, vol. 157, no. 5, pp. 934-940, 2007.

[7] M. Eshki, L. Allanore, P. Musette et al., "Twelve-year analysis of severe cases of drug reaction with eosinophilia and systemic symptoms: a cause of unpredictable multiorgan failure," Archives of Dermatology, vol. 145, no. 1, pp. 67-72, 2009.

[8] W.-H. Chung, S.-I. Hung, H.-S. Hong et al., "Medical genetics: a marker for Stevens-Johnson syndrome," Nature, vol. 428, no. 6982, p. 486, 2004.

[9] W.-H. Chung, S.-I. Hung, J.-Y. Yang et al., "Granulysin is a key mediator for disseminated keratinocyte death in StevensJohnson syndrome and toxic epidermal necrolysis," Nature Medicine, vol. 14, no. 12, pp. 1343-1350, 2008.

[10] S. G. Leckband, J. R. Kelsoe, H. M. Dunnenberger et al., "Clinical pharmacogenetics implementation consortium guidelines for HLA-B genotype and carbamazepine dosing," Clinical Pharmacology \& Therapeutics, vol. 94, no. 3, pp. 324-328, 2013.

[11] P. Chen, J.-J. Lin, C.-S. Lu et al., "Carbamazepine-induced toxic effects and HLA-B* 1502 screening in Taiwan," The New England Journal of Medicine, vol. 364, no. 12, pp. 1126-1133, 2011.
[12] W. M. Schoonen, S. L. Thomas, E. C. Somers et al., "Do selected drugs increase the risk of lupus? A matched case-control study," British Journal of Clinical Pharmacology, vol. 70, no. 4, pp. 588596, 2010.

[13] S.-I. Hung, W.-H. Chung, S.-H. Jee et al., "Genetic susceptibility to carbamazepine-induced cutaneous adverse drug reactions," Pharmacogenetics and Genomics, vol. 16, no. 4, pp. 297-306, 2006.

[14] C. B. Man, P. Kwan, L. Baum et al., "Association between HLA$\mathrm{B}^{*} 1502$ allele and antiepileptic drugs-induced cutaneous reactions in Han Chinese," Epilepsia, vol. 48, no. 5, pp. 1015-1018, 2007.

[15] W.-H. Chung and S.-I. Hung, "Recent advances in the genetics and immunology of Stevens-Johnson syndrome and toxic epidermal necrosis," Journal of Dermatological Science, vol. 66, no. 3, pp. 190-196, 2012.

[16] V. L. Yip, A. G. Marson, A. L. Jorgensen, M. Pirmohamed, and A. Alfirevic, "HLA genotype and carbamazepine-induced cutaneous adverse drug reactions: a systematic review," Clinical Pharmacology \& Therapeutics, vol. 92, no. 6, pp. 757-765, 2012.

[17] W. Tangamornsuksan, N. Chaiyakunapruk, R. Somkrua, M. Lohitnavy, and W. Tassaneeyakul, "Relationship between the $H L A-B^{*} 1502$ allele and carbamazepine-induced StevensJohnson syndrome and toxic epidermal necrolysis: a systematic review and meta-analysis," JAMA Dermatology, vol. 149, no. 9, pp. 1025-1032, 2013.

[18] T. Ozeki, T. Mushiroda, A. Yowang et al., "Genome-wide association study identifies $H L A-A^{*} 3101$ allele as a genetic risk factor for carbamazepine-induced cutaneous adverse drug reactions in Japanese population," Human Molecular Genetics, vol. 20, no. 5, pp. 1034-1041, 2011.

[19] M. McCormack, A. Alfirevic, S. Bourgeois et al., "HLA-A*3101 and carbamazepine-induced hypersensitivity reactions in Europeans," The New England Journal of Medicine, vol. 364, no. 12, pp. 1134-1143, 2011.

[20] E. Genin, D. P. Chen, S. I. Hung et al., "HLA-A*31: 01 and different types of carbamazepine-induced severe cutaneous adverse reactions: an international study and meta-analysis," The Pharmacogemonics Journal, 2013.

[21] Y.-H. Hsiao, R. C.-Y. Hui, T. Wu et al., "Genotype-phenotype association between HLA and carbamazepine-induced hypersensitivity reactions: strength and clinical correlations," Journal of Dermatological Science, vol. 73, no. 2, pp. 101-109, 2014.

[22] S.-H. Kim, K. W. Lee, W.-J. Song et al., "Carbamazepine-induced severe cutaneous adverse reactions and HLA genotypes in Koreans," Epilepsy Research, vol. 97, no. 1, pp. 190-197, 2011.

[23] N. Kaniwa, Y. Saito, M. Aihara et al., "HLA-B*1511 is a risk factor for carbamazepine-induced Stevens-Johnson syndrome and toxic epidermal necrolysis in Japanese patients," Epilepsia, vol. 51, no. 12, pp. 2461-2465, 2010.

[24] Y.-W. Shi, F.-L. Min, B. Qin et al., "Association between HLA and Stevens-Johnson syndrome induced by carbamazepine in Southern Han Chinese: genetic markers besides B* 1502 ?" Basic \& Clinical Pharmacology \& Toxicology, vol. 111, no. 1, pp. 58-64, 2012.

[25] H. Ikeda, Y. Takahashi, E. Yamazaki et al., "HLA class I markers in Japanese patients with carbamazepine-induced cutaneous adverse reactions," Epilepsia, vol. 51, no. 2, pp. 297-300, 2010.

[26] C.-Y. Wei, W.-H. Chung, H.-W. Huang, Y.-T. Chen, and S.-I. Hung, "Direct interaction between HLA-B and carbamazepine activates T cells in patients with Stevens-Johnson syndrome," 
The Journal of Allergy and Clinical Immunology, vol. 129, no. 6, pp. 1562-1569.e5, 2012.

[27] Y.-K. Cheung, S.-H. Cheng, E. J. M. Chan, S. V. Lo, M. H. L. Ng, and P. Kwan, "HLA-B alleles associated with severe cutaneous reactions to antiepileptic drugs in Han Chinese," Epilepsia, vol. 54, no. 7, pp. 1307-1314, 2013.

[28] A. Alfirevic, A. L. Jorgensen, P. R. Williamson, D. W. Chadwick, B. K. Park, and M. Pirmohamed, "HLA-B locus in Caucasian patients with carbamazepine hypersensitivity," Pharmacogenomics, vol. 7, no. 6, pp. 813-818, 2006.

[29] S.-C. Su and W.-H. Chung, "Update on pathobiology in StevensJohnson syndrome and toxic epidermal necrolysis," Dermatologica Sinica, vol. 31, no. 4, pp. 175-180, 2013.

[30] S.-I. Hung, W.-H. Chung, Z.-S. Liu et al., "Common risk allele in aromatic antiepileptic-drug induced Stevens-Johnson syndrome and toxic epidermal necrolysis in Han Chinese," Pharmacogenomics, vol. 11, no. 3, pp. 349-356, 2010.

[31] C. Locharernkul, J. Loplumlert, C. Limotai et al., "Carbamazepine and phenytoin induced Stevens-Johnson syndrome is associated with HLA-B* 1502 allele in Thai population," Epilepsia, vol. 49, no. 12, pp. 2087-2091, 2008.

[32] M. S. Hershfield, J. T. Callaghan, W. Tassanccyakul et al., "Clinical pharmacogenetics implementation consortium guidelines for human leukocyte antigen-B genotype and allopurinol dosing," Clinical Pharmacology \& Therapeutics, vol. 93, no. 2, pp. 153-158, 2013.

[33] S.-L. Hung, W.-H. Chung, L.-B. Liou et al., "HLA-B*5801 allele as a genetic marker for severe cutaneous adverse reactions caused by allopurinol," Proceedings of the National Academy of Sciences of the United States of America, vol. 102, no. 11, pp. 41344139, 2005.

[34] R. Somkrua, E. E. Eickman, S. Saokaew, M. Lohitnavy, and N. Chaiyakunapruk, "Association of HLA-B*5801 allele and allopurinol-induced stevens johnson syndrome and toxic epidermal necrolysis: a systematic review and meta-analysis," $B M C$ Medical Genetics, vol. 12, article 118, 2011.

[35] M. Lee, Y. Chen, and C. A. Shen, "A prospective study of HLA$\mathrm{B}^{*} 5801$ genotyping for the prevention of allopurinol induced severe cutaneous adverse reactions," in Proceedings of the113th Annual Meeting of the American Society for Clinical Pharmacology and Therapeutics, abstract S107, National Harbor, Maryland, March 2012.

[36] W. Tassaneeyakul, T. Jantararoungtong, P. Chen et al., "Strong association between HLA-B*5801 and allopurinol-induced Stevens-Johnson syndrome and toxic epidermal necrolysis in a Thai population," Pharmacogenetics and Genomics, vol. 19, no. 9, pp. 704-709, 2009.

[37] Z.-H. Cao, Z.-Y. Wei, Q.-Y. Zhu et al., "HLA-B* 58:01 allele is associated with augmented risk for both mild and severe cutaneous adverse reactions induced by allopurinol in Han Chinese," Pharmacogenomics, vol. 13, no. 10, pp. 1193-1201, 2012.

[38] M. H. Lee, S. L. Stocker, J. Anderson et al., "Initiating allopurinol therapy: do we need to know the patient's human leucocyte antigen status?" Internal Medicine Journal, vol. 42, no. 4, pp. 411416, 2012.

[39] D. Khanna, J. D. Fitzgerald, P. P. Khanna et al., "2012 American College of Rheumatology guidelines for management of gout. Part 1: systematic nonpharmacologic and pharmacologic therapeutic approaches to hyperuricemia," Arthritis Care \& Research, vol. 64, no. 10, pp. 1431-1446, 2012.
[40] S. Chohan, "Safety and efficacy of febuxostat treatment in subjects with gout and severe allopurinol adverse reactions," The Journal of Rheumatology, vol. 38, no. 9, pp. 1957-1959, 2011.

[41] J. S. Sundy, H. S. Baraf, R. A. Yood et al., "Efficacy and tolerability of pegloticase for the treatment of chronic gout in patients refractory to conventional treatment: two randomized controlled trials," The Journal of the American Medical Association, vol. 306, no. 7, pp. 711-720, 2011.

[42] J.-W. Jung, W.-J. Song, Y.-S. Kim et al., "HLA-B58 can help the clinical decision on starting allopurinol in patients with chronic renal insufficiency," Nephrology Dialysis Transplantation, vol. 26, no. 11, pp. 3567-3572, 2011.

[43] S. Hetherington, S. McGuirk, G. Powell et al., "Hypersensitivity reactions during therapy with the nucleoside reverse transcriptase inhibitor abacavir," Clinical Therapeutics, vol. 23, no. 10, pp. 1603-1614, 2001.

[44] M. Shapiro, K. M. Ward, and J. J. Stern, "A near-fatal hypersensitivity reaction to abacavir: case report and literature review," AIDS Reader, vol. 11, no. 4, pp. 222-226, 2001.

[45] P. Bossi, J. C. Roujeau, F. Bricaire, and E. Caumes, "StevensJohnson syndrome associated with abacavir therapy," Clinical Infectious Diseases, vol. 35, no. 7, p. 902, 2002.

[46] R. Pahk, M. C. Azu, B. R. Taira, and S. Sandoval, "Antiretroviralinduced toxic epidermal necrolysis in a patient positive for human immunodeficiency virus," Clinical and Experimental Dermatology, vol. 34, no. 8, pp. e775-e777, 2009.

[47] S. Hetherington, A. R. Hughes, M. Mosteller et al., "Genetic variations in $H L A-B$ region and hypersensitivity reactions to abacavir," The Lancet, vol. 359, no. 9312, pp. 1121-1122, 2002.

[48] S. Mallal, D. Nolan, C. Witt et al., "Association between presence of $H L A-B^{*} 5701, H L A-D R 7$, and HLA-DQ3 and hypersensitivity to HIV-1 reverse-transcriptase inhibitor abacavir," The Lancet, vol. 359, no. 9308, pp. 727-732, 2002.

[49] H. Gatanaga, H. Honda, and S. Oka, "Pharmacogenetic information derived from analysis of HLA alleles," Pharmacogenomics, vol. 9, no. 2, pp. 207-214, 2008.

[50] A. R. Hughes, M. Mosteller, A. T. Bansal et al., "Association of genetic variations in HLA-B region with hypersensitivity to abacavir in some, but not all, populations," Pharmacogenomics, vol. 5, no. 2, pp. 203-211, 2004.

[51] S. Mallal, E. Phillips, G. Carosi et al., "HLA-B*5701 screening for hypersensitivity to abacavir," The New England Journal of Medicine, vol. 358, no. 6, pp. 568-579, 2008.

[52] E. J. Phillips, J. R. Sullivan, S. R. Knowles, and N. H. Shear, "Utility of patch testing in patients with hypersensitivity syndromes associated with abacavir," AIDS, vol. 16, no. 16, pp. 2223-2225, 2002.

[53] E. J. Phillips, G. A. Wong, R. Kaul et al., "Clinical and immunogenetic correlates of abacavir hypersensitivity," AIDS, vol. 19, no. 9, pp. 979-981, 2005.

[54] N. H. Shear, B. Milpied, D. P. Bruynzeel, and E. J. Phillips, "A review of drug patch testing and implications for HIV clinicians," AIDS, vol. 22, no. 9, pp. 999-1007, 2008.

[55] E. J. Phillips and S. A. Mallal, "Pharmacogenetics of drug hypersensitivity," Pharmacogenomics, vol. 11, no. 7, pp. 973-987, 2010.

[56] A. M. Martin, D. Nolan, I. James et al., "Predisposition to nevirapine hypersensitivity associated with HLA-DRB1 ${ }^{*} 0101$ and abrogated by low CD4 T-cell counts," AIDS, vol. 19, no. 1, pp. 97-99, 2005. 
[57] S. Chantarangsu, T. Mushiroda, S. Mahasirimongkol et al., "HLA-B*3505 allele is a strong predictor for nevirapineinduced skin adverse drug reactions in HIV-infected Thai patients," Pharmacogenetics and Genomics, vol. 19, no. 2, pp. 139-146, 2009.

[58] R. Littera, C. Carcassi, A. Masala et al., "HLA-dependent hypersensitivity to nevirapine in Sardinian HIV patients," AIDS, vol. 20, no. 12, pp. 1621-1626, 2006.

[59] H. Gatanaga, H. Yazaki, J. Tanuma et al., "HLA-Cw8 primarily associated with hypersensitivity to nevirapine," AIDS, vol. 21, no. 2, pp. 264-265, 2007.

[60] C. Ciccacci, D. di Fusco, M. C. Marazzi et al., "Association between CYP2B6 polymorphisms and nevirapine-induced SJS/ TEN: a pharmacogenetics study," European Journal of Clinical Pharmacology, vol. 69, no. 11, pp. 1909-1916, 2013.

[61] F.-R. Zhang, H. Liu, A. Irwanto et al., " $H L A-B^{*} 13: 01$ and the dapsone hypersensitivity syndrome," The New England Journal of Medicine, vol. 369, no. 17, pp. 1620-1628, 2013.

[62] J. C. Sacco, M. Abouraya, A. Motsinger-Reif, S. H. Yale, C. A. McCarty, and L. A. Trepanier, "Evaluation of polymorphisms in the sulfonamide detoxification genes NAT2, CYB5A, and CYB5R3 in patients with sulfonamide hypersensitivity," Pharmacogenetics and Genomics, vol. 22, no. 10, pp. 733-740, 2012.

[63] A.-Y. Lee, M.-J. Kim, W.-Y. Chey, J. Choi, and B.-G. Kim, "Genetic polymorphism of cytochrome $P_{450} 2 \mathrm{C} 9$ in diphenylhydantoin-induced cutaneous adverse drug reactions," European Journal of Clinical Pharmacology, vol. 60, no. 3, pp. 155-159, 2004. 


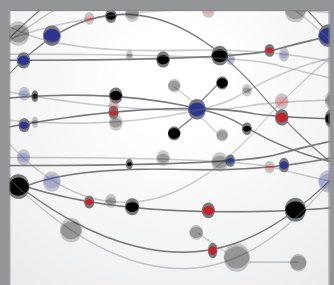

The Scientific World Journal
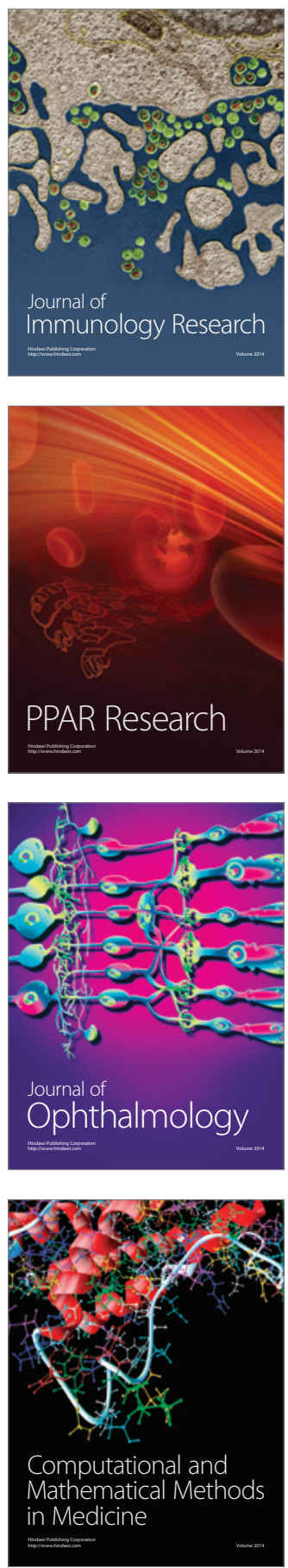

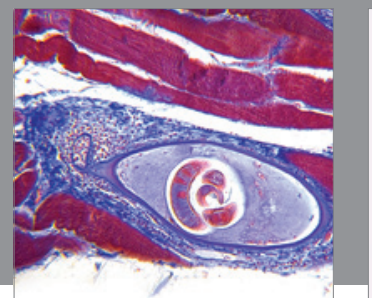

Gastroenterology

Research and Practice
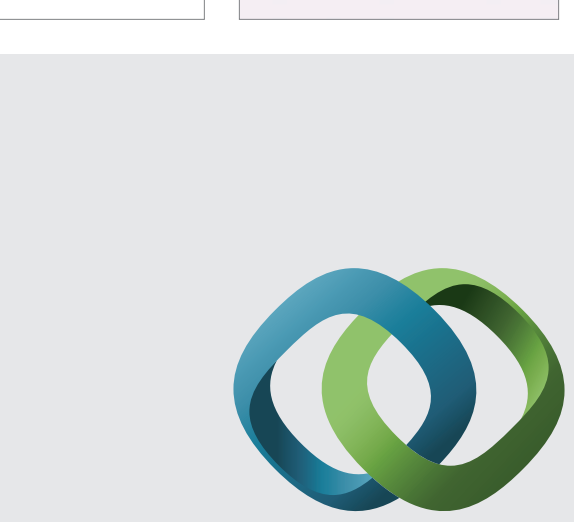

\section{Hindawi}

Submit your manuscripts at

http://www.hindawi.com
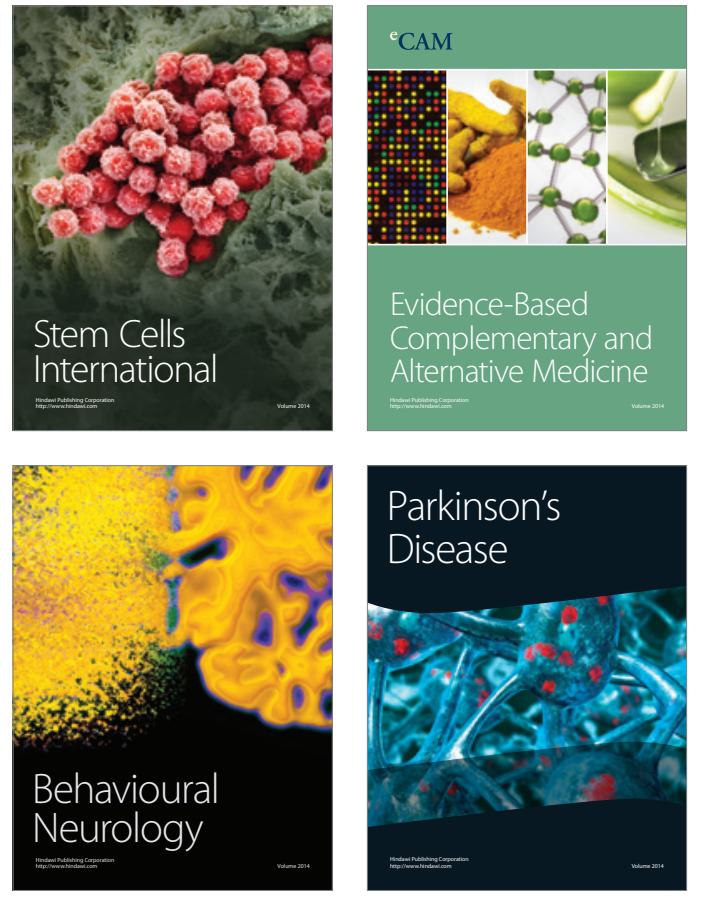
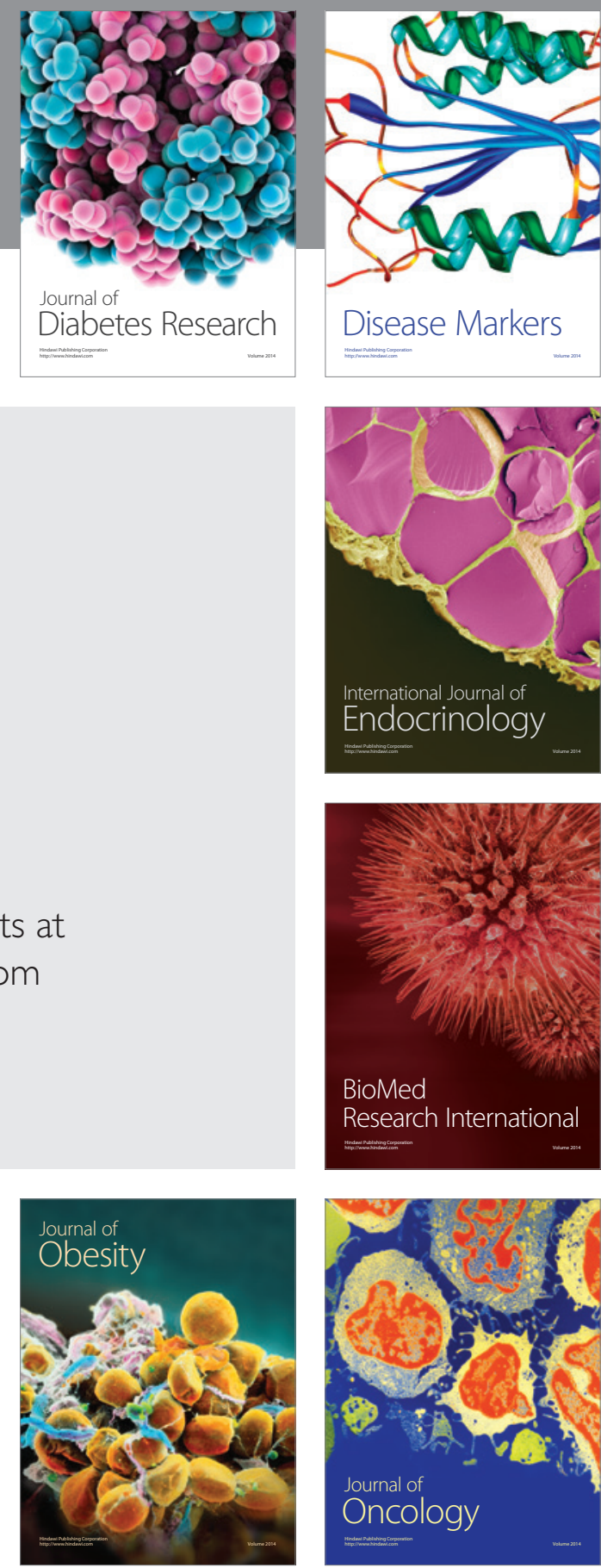

Disease Markers
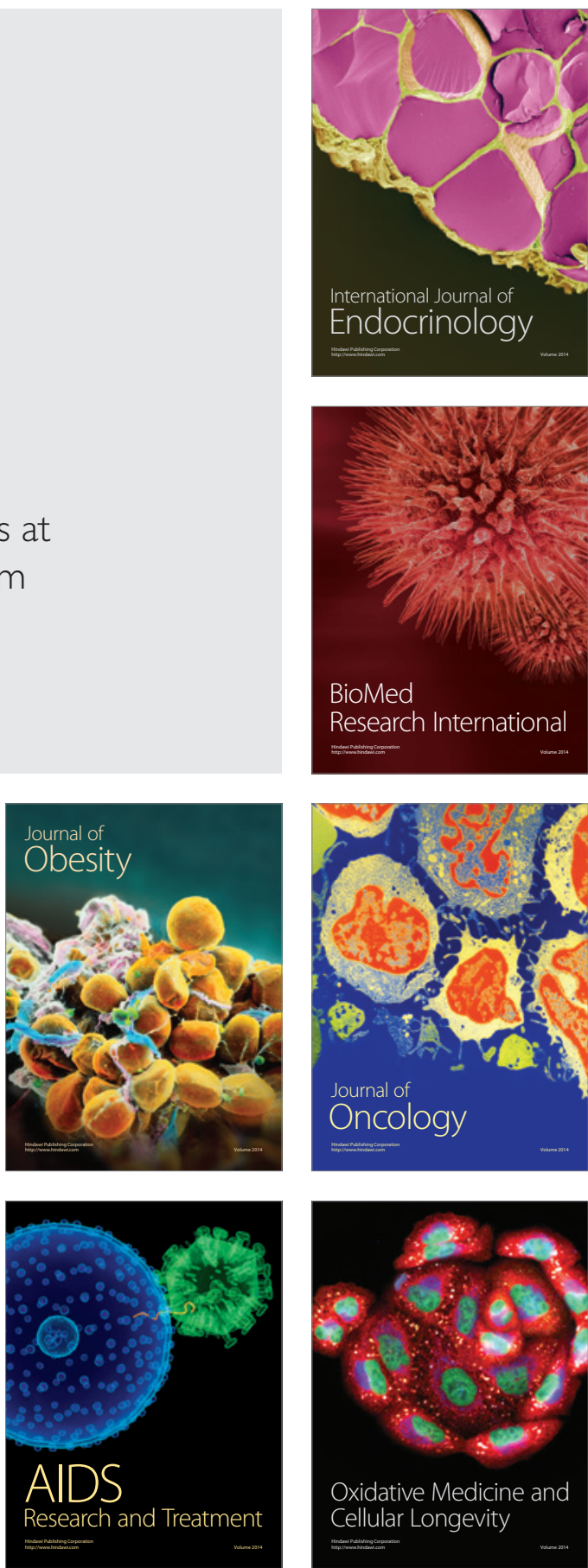